\title{
Integrative meta-analysis of protein interaction data identified multiple GID/MRCTLH protein complexes in plants
}

\author{
Mercè Miquel, Carlos Vicient* \\ Centre for Research in Agricultural Genomics (Consejo Superior de Investigaciones Científicas-Institut de \\ Recerca i Tecnologia Agroalimentàries-Universitat Autònoma de Barcelona-Universitat de Barcelona), Campus \\ UAB, Bellaterra (Cerdanyola del Vallès), 08193 Barcelona, Spain
}

*Corresponding author: carlos.vicient@cragenomica.es

\begin{abstract}
GID/MRCTLH is a protein complex involved in the regulation of several cellular processes through the polyubiquitination and proteosome degradation. It has been described in yeast and mammals. Genes coding for homologous proteins are also present in plant genomes but have been little studied. BLAST analyses revealed that genes coding for members of the GID/MRCTLH complex are found in multiple copies in plants, compared to mammals and yeast. The potential structure of the Arabidopsis GID/MRCTLH complex was estimated based on the Arabidopsis protein interaction database Interactome 2.0. According to these data, Arabidopsis may contain two GID/MRCTLH complexes instead of the one described in yeast and mammals. The structure of the two Arabidopsis complexes seem to be similar to the yeast GID complex, and seem to interact with several other proteins out of the complex. These data suggest that, similarly to yeast and mammals, the plant GID/MRCTLH complexes are involved in the regulation of several cellular processes through proteosome protein degradation.
\end{abstract}

Keywords: GID, MRCTLH, protein interaction, ubiquitination, Arabidopsis.

Abbreviations: CTLH_C-terminal LisH motif, CRA_CT11_RanBPM domain, GID_Glucose-Induced Degradation Complex, LisH_ LIS1 homology domain, MRCTLH_muskelin/RanBP9/CTLH complex.

\section{Introduction}

High-throughput "-omics" technologies have enabled global measurement of biological molecules (DNA, RNA, proteins, metabolites, etc.) and their interactions, producing network modules. Among different molecular networks, proteinprotein interaction networks derived from small- to largescale experiments have emerged as an important resource for understanding essential biological processes (Tong et al., 2002). The first step required to perform protein-protein interaction network analysis is to identify the proteins of interest. The second step is to use the identified proteins to search and retrieve binary interactions from a curated proteinprotein interaction databases. A network can be assembled based on the set of interactions.

The GID/MRCTLH protein complex was first discovered in yeast (S. cerevisiae), where it was named GID (glucoseinduced degradation deficient)(Regelmann et al., 2003). GID complex is a $600 \mathrm{kDa}$ assembly of seven proteins (GID1 (VID30), GID2, GID4 (VID24), GID5 (VID28), GID7, GID8 and GID9). GID functions as an ubiquitin-ligase complex that targets fructose-1,6-bisphosphatase and other key gluconeogenic enzymes for polyubiquitination and subsequent proteasomal degradation when cells are supplied with glucose (Francis et al., 2013; Santt et al., 2008). Many of the proteins in the GID complex contain LisH and CTLH domains.

The GID complex has a counterpart in mammals where it is named MRCTLH (muskelin/RanBP9/CTLH). MRCTLH contains proteins that are homologous in domain organisation and sequence identity to the yeast GID proteins, including
RanBP9 (RanBPM), Rmnd5a, c17orf39, ARMc8, WDR26, TWA1 and MAEA (Francis et al., 2013)(Table 1). The mammalian MRCTLH complex also has E3 ubiquitin ligase activity. In humans, components of the CTLH complex are involved in a variety of processes as the promotion of the interaction of hepatocyte growth factor-regulated tyrosine kinase substrate with ubiquitinated proteins (Tomaru et al., 2010), the regulation of the degradation of the alpha-catenin (Suzuki et al., 2008), the nuclear extrusion in erythropoeisis (Soni et al., 2006), the regulation of cell morphology (Valiyaveettil et al., 2008) or the regulation of the stability of different proteins (Kramer et al., 2005; Danserau et al., 2008; Suresh et al., 2010).

Plant genomes encode for proteins homologous to the members of GID/MRCTLH complex (Tomaštiková et al., 2012; Francis et al., 2013), suggesting that these complexes are also present in plants. Accordingly, the Arabidopsis RanBPM homologue is part of soluble cytoplasmic complexes of about $400 \mathrm{kDa}$ in size, complexes that also include proteins that are homologue to other components of the GID/MRCTLH complex in mammals and yeast (Tomaštíková et al., 2012). The role of the Arabidopsis CTLH complex is unknown.

A difference between plants and the other Eukaryotes is that plant genomes contain a higher number of genes coding for putative components of the GID/MRCTLH complex (Francis et al., 2013). In view of the potential fundamental roles of the GID/MRCTLH protein complexes in plants there is a great need for better understand the relationships of the 
protein components of the complex in plants. To this end we have identified the individual components of the GID/MRCTLH complex encoded by the Arabidopsis genome and we have examined their possible protein-protein interactions to determine the potential structure of the GID/MRCTLH complexes in plants and their potential roles.

\section{Results}

\section{Identification of MRCTLH components in Arabidopsis} thaliana

In order to identify the proteins in Arabidopsis thaliana which are homologues to the proteins in the GID/MRCTLH complex we searched by BLASTP method against the Arabidopsis protein sequences. We used as query the sequences of the proteins of the human MRCTLH complex (Table 1). Proteins having an e-value lower than 1e-19 were selected. The domain organization of the selected proteins was determined using SMART (Letunic et al., 2012). Only proteins having a similar protein domain organization as their human counterparts were selected. We finally identified 16 Arabidopsis proteins: 4 corresponding to RanBPM (Santt et al., 2008), 4 to TWA1 (Santt et al., 2008), one to MAEA (Tong et al., 2002), 3 to Rmd5 (Francis et al., 2013), 3 to WDR26 (Regelmann et al., 2003) and one to ARMC8 (Tong et al., 2002). We did not find homologues to c17ORF39 or muskelin.

For interactions of Arabidopsis proteins encoding for MRCTLH proteins researchers have made a systematic attempt to determine the possible interactions between the proteins encoded by Arabidopsis genome. The Arabidopsis Interactions Viewer (Geisler-Lee et al., 2007; http://bar.utoronto.ca/interactions/cgi-bin/arabidopsis_

interactions_viewer.cgi; Interactome 2.0) queries a database of 70944 predicted and 36329 confirmed Arabidopsis protein interactions. The confirmed interactions were determined using yeast two hybrid screens and traditional biochemical methods. Using this facility we determined all the possible protein interactions of the Arabidopsis proteins which are putative components of the GID/MRCTLH complex (Table 1) and we found 239 interactions involving 11 of the 16 proteins (Supplementary Table 1). These interactions include 2 self-interactions (AT5G09630 and AT4G37880), 27 interactions between putative components of the MRCTLH complex and 210 interactions with other proteins.

AT5G09630 and AT4G37880 are predicted to self-interact. Both proteins contain a LisH domain. LisH-dependent dimerization has been observed for several proteins (Kim et al., 2004; Mikolajka et al., 2006; Oberoi et al., 2011; Delto et al., 2015). This data suggests that the LisH domains encoded by AT5G09630 and AT4G37880 mediate their selfinteraction.

The domain organization of the protein encoded by At5g67320 gene is similar to the human WDR26, but the evalue of $7 \mathrm{e}-19$ prompted us to initially do not select it as a putative member of the plant CTLH complex (Table 1). However, AT5G67320 interacts with two of the predicted Arabidopsis MRCTLH proteins (AT4G09300 and AT2G22690), suggesting that, despite the low similarity, it is a component of the Arabidopsis MRCTLH complex.

Based on the data obtained from the protein-protein interaction database, we generated a model of the Arabidopsis GID/MRCTLH complex (Fig. 1A) and we compared it with the known structure of the yeast GID complex (Fig. 1B; Menssen et al., 2012). These model suggests the existence of two GID/MRCTLH complexes in Arabidopsis (A and B). The structure of the two putative Arabidopsis GID/MRCTLH complexes is similar, but not identical, to the structure of the yeast GID complex. For example:

- AT1G35470 (similar to RanBPM/GID1) is the only protein present in both complexes and occupies a central position as GID1 does in the GID complex.

- In yeast, GID8 is the second central component of the complex and is similar to human TWA1. AT1G61150 is similar to TWA1/GID8 and occupies a similar position in complex B. If we look at the same position in complex A, this position is occupied by AT3G55070, but AT3G55070 is similar to MAEA/GID9. However, the structure of the proteins AT3G55070/MAEA/GID9 is similar to the structure of proteins AT1G61150/TWA1/GID8, all them contain LisH, CTLH and CRA domains. Altogether suggests that, despite the differences in similarity, AT3G55070 plays a similar role as AT1G61150 in the core of the Arabidopsis GID/MRCTLH complex A.

- In yeast, GID7 binds GID1. AT5G08560 and AT5G43920 are similar to GID7/WDR26, and both are predicted to bind AT1G35470, which is similar to GID1/RanBPM. In consequence, AT5G08560 and AT5G43920 seem to occupy a similar position as GID7 in the complexes A and B, respectively.

- Three of the Arabidopsis selected proteins are similar to Rmd5/GID2 and also contain a RING domain. GID2 in yeast is located in the periphery of the complex, interacting with GID5 and GID9. AT2G22690 and AT5G09630 are located in a similar position in complex $\mathrm{A}$ and $\mathrm{B}$, respectively, suggesting they play a similar role. At4G37880 occupies in Arabidopsis Complex B a similar position as GID9 in yeast. The RING domain is a specialized type of Zn-finger that has E3 ubiquitin-protein ligase activity and determines the substrate specificity for ubiquitination (Stone et al., 2005). The fact that proteins containing RING domain are located in a similar position in yeast and in both Arabidopsis complexes suggests that they have a similar role in the determination of the substrate specificity for ubiquitination.

- AT5G67320 encodes a protein similar to GID7/WDR26 but is located in a very different position within the complex A compared to yeast. This position is similar to the occupied by GID4 and GID5 in yeast. However, homologues to GID4 and GID5 are absent in Arabidopsis. These data suggests that AT5G67320 plays a similar role as GID4 or GID5 despite their differences in protein structure. In humans, ARMc8 (homologue to GID5) occupies a similar position in the MRCTLH complex. ARMc8 plays a regulatory role. For example, ARMc8 associates with alpha-catenin and upregulates its degradation (Suzuki et al., 2008). Interestingly, AT5G67320 interacts with 78 proteins outside the complex, suggesting that it may play a similar regulatory function as ARMc8.

- AT4G09300 is similar to TWA1/GID8 and interacts with AT5G67320 (in complex A) and with AT5G09630 (in complex B). These data opens the possibility that complexes $\mathrm{A}$ and $\mathrm{B}$ correspond, in fact, to a single, unique, very big complex, bigger than the yeast GID and the mammal MRCTLH complexes. However, it was determined that AtRanBPM (AT1G35470) is located predominantly in the form of soluble cytoplasmic complexes of $\sim 230-500 \mathrm{kDa}$ in size (Tomaštíková et al., 2012), which may correspond to the size of complex A or the size of complex B, but not to the size of both together. In any case, at the present, we don't know the possible role of AT4G09300. 
Table 1. Arabidopsis thaliana proteins putative homologs of human MRCTLH components.

\begin{tabular}{|c|c|c|c|c|c|c|}
\hline Human & Domains (human) 1 & Yeast & Arabidopsis & E-value & Domains (Arabidopsis) 1 & Complex \\
\hline \multirow{4}{*}{$\begin{array}{l}\text { RanBPM } \\
\text { (RanBP9) }\end{array}$} & & \multirow{4}{*}{ GID1 } & At1G35470 & $8 \mathrm{e}-44$ & & A B \\
\hline & & & AT4G09340 & $6 e-36$ & & B \\
\hline & & & AT4G09200 & $8 \mathrm{e}-28$ & & - \\
\hline & & & AT4G09310 & $8 \mathrm{e}-28$ & & - \\
\hline \multirow{4}{*}{ TWA1 } & & \multirow{4}{*}{ GID8 } & AT1G61150 & $2 \mathrm{e}-56$ & & $\mathrm{~B}$ \\
\hline & & & AT4G09300 & $1 \mathrm{e}-37$ & & A \\
\hline & & & AT1G11110 & $7 e-28$ & & - \\
\hline & & & AT1G06060 & 7e-192 & & - \\
\hline MAEA & & GID9 & AT3G55070 & $2 \mathrm{e}-47$ & & A \\
\hline \multirow{3}{*}{ Rmd5 } & \multirow{6}{*}{$=-A$ стин $\rightarrow D$} & \multirow{3}{*}{ GID2 } & AT4G37880 & $1 \mathrm{e}-57$ & & B \\
\hline & & & AT2G22690 & $9 e-53$ & & A \\
\hline & & & AT5G09630 & $2 \mathrm{e}-47$ & & B \\
\hline \multirow{3}{*}{ WDR26 } & & & AT5G08560 & $4 \mathrm{e}-83$ & & A \\
\hline & & GID7 & AT5G43920 & $9 \mathrm{e}-80$ & & B \\
\hline & & & $\begin{array}{l}\text { AT5G67320 } \\
2\end{array}$ & - & & $t_{\mathrm{A}}$ \\
\hline ARMC8 & & GID5 & AT1G51350 & $4 e-38$ & -- & - \\
\hline c17ORF39 & 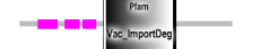 & GID4 & -- & --- & -- & - \\
\hline Muskelin 1 & 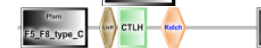 & - & - & -- & - & - \\
\hline
\end{tabular}

2 This protein was not initially selected because a high E-value but further analyses suggested that it is part of the complex.

A

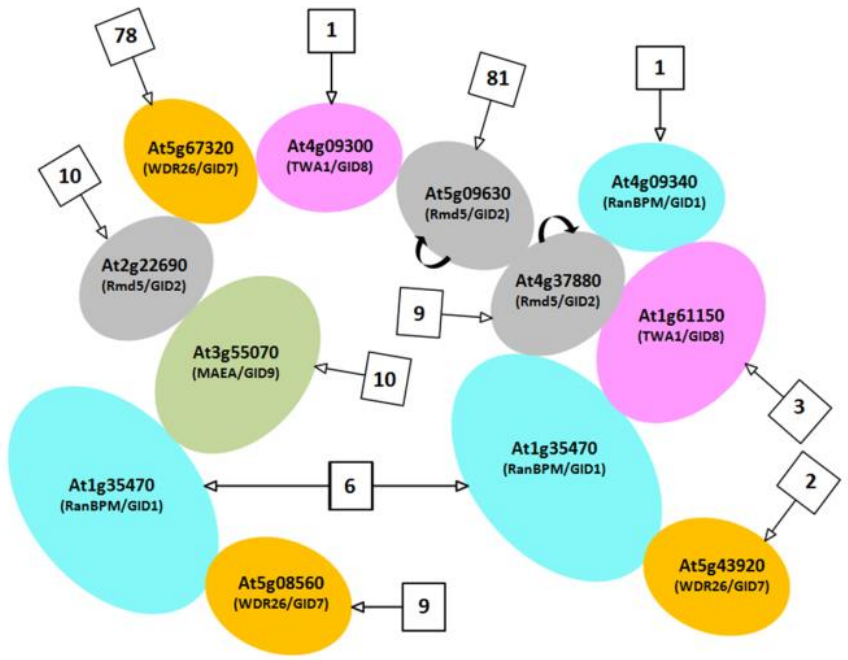

Complex A

Complex B

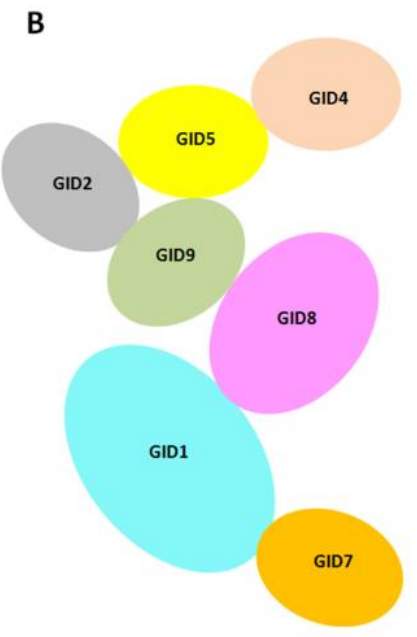

GID (yeast)

Fig 1. Schematic representation of the MRCTLH/GID protein complexes in Arabidopsis and yeast. (A) Structure of Arabidopsis MRCTLH/GID protein complexes according to the data in The Arabidopsis Interactions Viewer. (B) Structure of yeast GID complex (Menssen et al., 2012). 


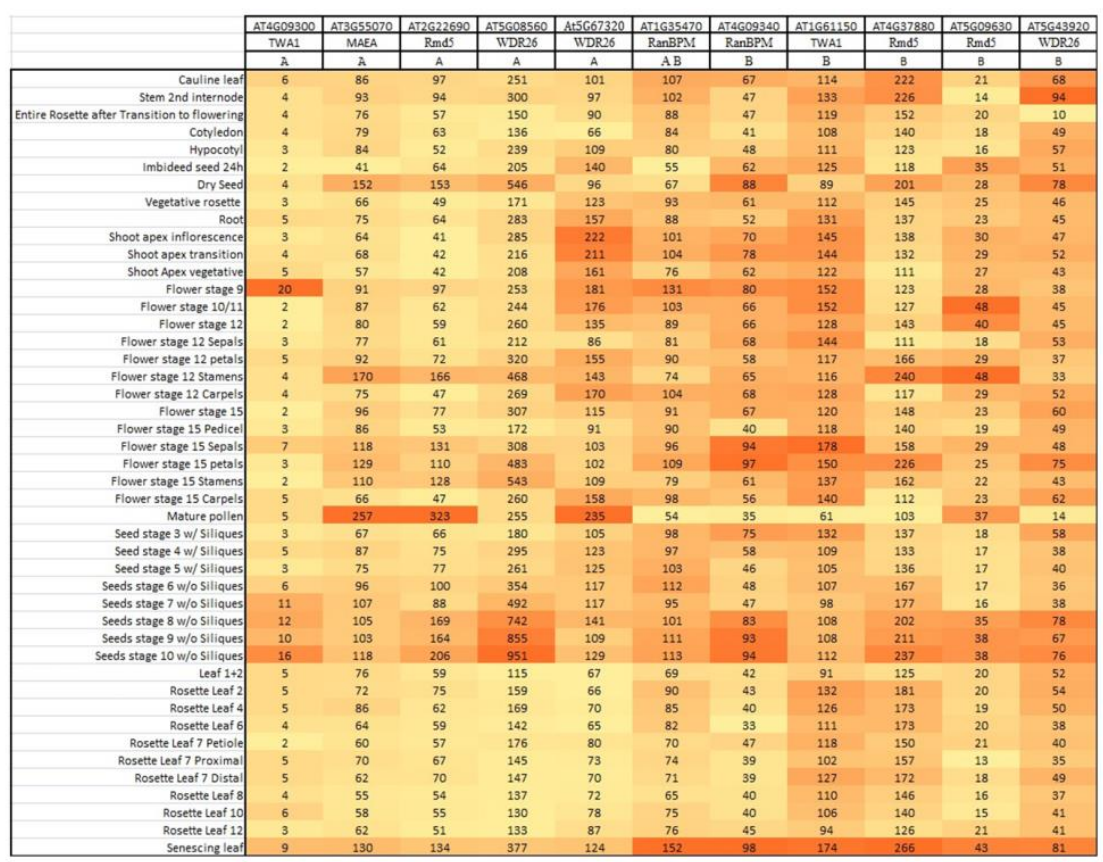

Fig 2. Transcription levels of the Arabidopsis genes coding for proteins in the GID/MRCTLH complex. The expression patterns of the Arabidopsis MRCTLH homologous genes were obtained in the Arabidopsis eFP Browser 2.0 (Winter et al., 2007) based on microarray hybridization data.

Expression patterns of Arabidopsis genes encoding for MRCTLH proteins

The expression patterns of the Arabidopsis MRCTLH homologous genes were obtained from the Arabidopsis eFP Browser 2.0 (Winter et al., 2007) based on microarray hybridization data. All the genes are, more or less, ubiquitously expressed in all the organs and developmental stages analysed (Fig. 2) with only some exceptions. At4g09300 and At5g09630 have a low level of expression comparing with the other genes except in mature flowers and pollen, and in seeds in late stages of development. Interestingly, the proteins coding by these genes are supposed to be located in the periphery of the complex (Fig, 1). In yeast GID this position is occupied by proteins that regulate the activity of the complex. For example, GID4, which occupies a similar position in GID complex and only binds the complex after glucose is added to the media and it might be a switch activating the GID complex to degrade FBPase (Santt et al., 2008).

All the other genes have a medium or high level of expression. In general, genes coding for proteins in complex $\mathrm{A}$ are expressed in lower levels than genes coding for proteins in complex B (Fig. 2). As mentioned, all the genes are expressed in all the plant organs examined, but their expression levels are higher in certain organs. For example, all the genes have a relative high expression in mature seeds and senescing leaves and genes in complex A also in pollen grains (mature flowers). These expression patterns are similar to the observed in the genes encoding for the MRCTLH components in humans, which are all widely expressed in multiple tissues (Francis et al., 2013).

Proteins interacting with the Arabidopsis GID/MRCTLH protein complexes 210 proteins are predicted to interact with the proteins in the complexes A and B (Fig. 1A, numbers in the squares; Supplementary Table 1): 114 with complex A and 104 with complex B. In special, AT5G67320 and AT5G09630 potentially interact with about 80 external proteins each. These numbers are similar to human and yeast where 208 and 310 interacting proteins have been described, respectively, according to BioGRID database (ChatrAryamontri et al., 2015; http://thebiogrid.org/).

The Arabidopsis interacting external proteins are involved in several different roles (Supplementary Table 1). Some differences were observed between interactors of complex A and interactors of complex B. Interactors of complex A are significantly more involved in the regulation of cell cycle, development and gene expression, in nuclear structure, in transport and in microtubules, whereas the interactors of complex B are especially involved in carbohydrate metabolism and regulation of ROS. These data suggest the existence of a certain specialization in the roles of both complexes.

One of the suggested roles of GID/MRCTLH complexes is the ubiquitin-ligase activity (Santt et al., 2008). In consequence, we examined how many of the Arabidopsis proteins interacting with the complexes have been demonstrated to be ubiquitinated. $19.8 \%$ of the proteins have been demonstrated to interact with polyubiquitin 3 (UBQ3; AT5G03240). UBQ3 have been demonstrated to interact with 1316 Arabidopsis proteins. Considering that the Arabidopsis genome contains about 27000 protein coding genes that means that $4.9 \%$ of the Arabidopsis proteins interact with UBQ3. These data means that proteins interacting with GID/MRCTLH complexes are four times more frequently ubiquitinated than the total Arabidopsis proteins, suggesting that GID/MRCTLH complexes in Arabidopsis are involved in protein ubiquitination.

\section{Discussion}

Although genes coding for homologous proteins of the MRCTLH/GID complex are present in the genomes of all plant species very little is known about this protein complex in plants (Francis et al., 2013). AtRanBPM (AT1G35470) is 
known to be part of a protein complex of about $500 \mathrm{kDa}$ (Tomaštíková et al., 2012) and immunoprecipitation of AtRamPBM identified different proteins similar to other components of the MRCTLH complex (Francis et al., 2013), suggesting that MRCTLH complexes also exist in Arabidopsis.

Compared with fungi or animal genomes, plant genomes contain more genes coding for MRCTLH proteins (Francis et al., 2013). A higher number of genes may suggest the existence of more than one complex in plants. The data presented here supports this idea. Two MCRTLH complexes seem to exist in Arabidopsis and they seem to have at least partially different roles because the roles of the external proteins that interact with them are significantly different. The functions of the GID/MRCTLH complex in the different species are poorly understood. In yeast, GID complex plays a ubiquitin ligase activity that is involved in proteasomal degradation of, at least, fructose-1,6-bisphosphatase (FBPase) and phosphoenolpyruvate carboxykinase during the transition from gluconeogenic to glycolytic growth conditions (Regelmann et al., 2003; Santt et al., 2008).

Some of the subunits of the mammals MRCTLH complex have been involved in different functions which need of protein-protein interactions. This is not surprising because most of the components contain LisH and CTLH protein domains that are known to mediate protein-protein interactions (Salemi et al., 2015). For example, to date, more than 50 protein interactions have been demonstrated for RanBPM (Francis et al., 2013; Suresh et al., 2012; Kobayashi et al., 2007). These proteins are involved in a variety of cellular processes, including cell adhesion and migration, microtubule dynamics, neuronal morphogenesis, male and female gametogenesis, gene transcription, regulation of protein stability, cell cycle regulation and other undefined cellular processes.

The components of Arabidopsis GID/MRCTLH complexes also interact with many other proteins and, in many cases, the interactors play similar roles as the observed in mammalian MRCTLH complex. For example:

- The mammalian MRCTLH complex has been involved in the proteasome-dependent degradation of non-ubiquitinated $\alpha$-catenin (Suzuki et al., 2008) and in the modulation of the endosome/lysosome-dependent degradation of ubiquitinated proteins via interaction with HRS (hepatocyte growth factorregulated tyrosine kinase substrate) (Tomaru et al., 2010). On the other hand, binding of ARMc8 up-regulates the proteasome-dependent degradation of $\alpha$-catenin (Suzuki et al., 2008). RanBPM interacts with the deubiquitinating enzyme (DUB) USP11 (Ideguchi et al., 2002). In Arabidopsis, different components of the GID/MRCHTL complexes interact with proteosome and ubiquitinationrelated proteins as, for example, $26 \mathrm{~S}$ proteasome AAAATPase subunit RPT5B, Ubiquitin-specific protease 9, Seven in Absentia of Arabidopsis 2, SKP-Like2, etc.

- The human RanBPM was first identified as a Ran binding protein (Nakamura et al., 1998). Ran (RAs-related Nuclear protein) is a small $G$ protein that is essential for the translocation of RNA and proteins through the nuclear pore complex (Moore et al., 1994). The Ran protein is also involved in the control of DNA synthesis and cell cycle progression, and mutations in Ran have been found to disrupt DNA synthesis (Sazer and Dasso, 2000). In Arabidopsis, the RanBPM homologue AT1G35470 interacts with RAN2 and RAN3, and other plant GID/MRCTLH proteins interact with different importins and the Nuclear Transport Factor 2B.

- Different plant GID/MRCTLH proteins interact with proteins involved in carbohydrate metabolism as hexokinase
2 and trehalose-6-phosphatase synthases, glucose-6phosphate acetyltransferase 1, galactose oxidase or ATSNF4, homolog of SNF4 (Yeast Sucrose Non-Fermenting 4). Interestingly, GID1 is required for rapamycin-induced degradation of the high affinity hexose transporter Hxt7 (Snowdon et al., 2008).

- Most of the yeast GID proteins are also involved in the vacuolar-dependent degradation of FBPase: GID1, GID4 and GID5 (Chiang et al., 1998). The vacuole dependent degradation pathway requires heat shock Ssa2, cyclophylin A and Vid22 (Chiang et al., 1998; Shieh et al., 2001). In mammal cells, ArMc8 was found to interact with the endosomal protein HRS hepatocyte growth factor regulated tyrosine kinase substrate which plays a role in endosomal trafficking of membrane proteins (Tomaru et al., 2010). In Arabidopsis, AT5G67320 (WDR26) interacts with Cyclophylin 19, AT2G22690 (Rmd5) interacts with Heat shock protein 83, and AT5G09630 (Rmd5) interacts with Autophagy18A.

- RanBPM was found to interact with several transcriptional factors and affecting their activity or stability (Atabakhsh et al., 2009; Atabakhsh et al., 2012). At least six transcription factors interact with Arabidopsis GID/MRCTLH proteins (DEK3, EMB1135, ATSWC2, EBS and E2F).

- RanBPM interacts with the cytoplasmatic domains of a variety of membrane receptors such as integrin $\beta$ subunit (Denti et al., 2004), c-Met (Wang et al., 2002), LICAM (Cheng et al., 2005), CD39 (Wu et al., 2006) and the calcium channel Cav.3.1. (Kim et al., 2009) to have a major role in regulating cell shape, arrangement, polarity or cell proliferation. AT5G09630 (Rmd5) interacts with a Lung seven transmembrane receptor family protein and a Membrane anchored Myb protein.

\section{Materials and Methods}

\section{Selection of proteins for analysis}

Arabidopsis orthologues of MRCTLH proteins were identified through BLASTp searches with the human MRCTLH components: RanBPM/RanBP9 (Q96S59/NP_005484), TWA1 (Q9NWU2/NP_060366), MAEA (Q7L5Y9/NP_001017405), Rmnd5A (Q9H871/NP 073617), WDR26 (NP 079436), ARMC8 (NP_056211), c17ORF39 (NP_076957) and Muskelin (Q9UL63/NP_037387). Searches were run against the nonredundant databases of the National Center for Biotechnology Information (NCBI) at default parameters (expect threshold of 10, word size of 6, BLOSUM62 matrix, gap cost existence of 11 and gap cost extension of 1 ).

\section{Determination of protein structure}

SMART (Simple Modular Architecture Research Tool; Letunic et al., 2012; http://smart.emblheidelberg. de/) was used to identify protein domains under standart conditions and to prepare logos for each protein.

\section{Determination of protein interactions}

Search for putative interacting proteins was done using The Arabidopsis Interactions Viewer (Geisler-Lee et al., 2007; http://bar.utoronto.ca/interactions/cgi-bin/arabidopsis_ interactions_viewer.cgi) based on the interactome v2.0 database using standart parameters. 


\section{Determination of gene expression}

The gene expression patterns were obtained from the Arabidopsis eFP Browser 2.0 (Winter et al., 2007; http://bar.utoronto.ca/efp2/Arabidopsis/Arabidopsis_eFPBro wser2.html).

\section{Conclusion}

Arabidopsis contain two different GID/MRCTLH complexes showing a structure which is similar to the yeast GID complex. Their components, structure and interactions with other proteins suggest that, similarly to GID/MRCTLH complexes in mammals and yeast, they also are involved in the regulation of several cellular processes through proteosome protein degradation.

\section{Acknowledgments}

This work was supported by grants from the Spanish Government (BIO2001-1721) and the Generalitat of Catalunya (2014SGR-1434).

\section{References}

Atabakhsh E, Bryce DM, Lefebvre KJ, Schild-Poulter C (2009) RanBPM has proapoptotic activities that regulate cell death pathways in response to DNA damage. Mol Cancer Res. 7:1962-1972.

Atabakhsh E, Wang JH, Wang X, Carter DE, Schild-Poulter C (2012) RanBPM expression regulates transcriptional pathways involved in development and tumorigenesis. Am J Cancer Res. 2:549-565.

Chatr-Aryamontri A, Breitkreutz BJ, Oughtred R, Boucher L, Heinicke S, Chen D, Stark C, Breitkreutz A, Kolas N, O'Donnell L, Reguly T, Nixon J, Ramage L, Winter A, Sellam A, Chang C, Hirschman J, Theesfeld C, Rust J, Livstone MS, Dolinski K, Tyers M (2015) The BioGRID interaction database: 2015 update. Nucleic Acids Res. 43:D470-478.

Cheng L, Lemmon S, Lemmon Y (2005) RanBPM is an L1interacting protein that regulates L1-mediated mitogenactivated protein kinase activation. J Neurochem. 94:11021110.

Chiang MC, Chiang HL (1998) Vid24p, a novel protein localized to the fructose-1, 6-bisphosphatase-containing vesicles, regulates targeting of fructose-1,6-bisphosphatase from the vesicles to the vacuole for degradation. J Cell Biol. 140:1347-1356.

Dansereau DA, Lasko P (2008) RanBPM regulates cell shape, arrangement, and capacity of the female germline stem cell niche in Drosophila melanogaster. J Cell Biol. 182:963-977.

Delto CF, Heisler FF, Kuper J, Sander B, Kneussel M, Schindelin H (2015) The LisH motif of muskelin is crucial for oligomerization and governs intracellular localization. Structure. 23:364-373.

Denti S, Simi A, Cheli A, Rogge L, Innamorati G, Putignano S, Fabbri M, Pardi R, Bianchi E (2004) RanBPM is a phosphoprotein that associates with the plasma membrane and interacts with the integrin LFA-1. J Biol Chem. 279:13027-13034.

Francis O, Han F, Adams JC (2013) Molecular phylogeny of a RING E3 ubiquitin ligase, conserved in eukaryotic cells and dominated by homologous components, the muskelin/RanBPM/CTLH complex. PLoS One 8:e75217.
Geisler-Lee J, O'Toole N, Ammar R, Provart NJ, Harvey Millar A, Geisler M (2007) A Predicted Interactome for Arabidopsis. Plant Physiol. 45:317-329.

Ideguchi $\mathrm{H}$, Ueda A, Tanaka M, Yang J, Tsuji T, Ohno S, Hagiwara E, Aoki A, Ishigatsubo Y (2002) Structural and functional characterization of the USP11 deubiquitinating enzyme, which interacts with the RanGTP-associated protein RanBPM. Biochem J. 367:87-95.

Kim MH, Cooper DR, Oleksy A, Devedjiev Y, Derewenda U, Reiner O, Otlewski J, Derewenda ZS (2004) The structure of the N-terminal domain of the product of the lissencephaly gene Lis1 and its functional implications. Structure 12:987-998.

Kim T, Kim S, Yun HM, Chung KC, Han YS, Shin HS, Rhim H (2009) Modulation of $\mathrm{Ca}(\mathrm{v}) 3.1$ T-type $\mathrm{Ca} 2+$ channels by the Ran-binding protein RanBPM. Biochem Bioph Res Co. 378:15-20.

Kobayashi N, Yang J, Ueda A, Suzuki T, Tomaru K, Takeno M, Okuda K, Ishigatsubo Y (2007) RanBPM, Muskelin, p48EMLP, p44CTLH, and the armadillo-repeat proteins ARMC8alpha and ARMC8beta are components of the CTLH complex. Gene 396:236-247.

Kramer S, Ozaki T, Miyazaki K, Kato C, Hanamoto T, Nakagawara A (2005) Protein stability and function of p73 are modulated by a physical interaction with RanBPM in mammalian cultured cells. Oncogene 24:938-944.

Letunic I, Doerks T, Bork P (2012) SMART 7: recent updates to the protein domain annotation resource. Nucleic Acids Res. 40:D302-305.

Menssen R, Schweiggert J, Schreiner J, Kusevic D, Reuther J, Braun B, Wolf DH (2012) Exploring the topology of the Gid complex, the E3 ubiquitin ligase involved in cataboliteinduced degradation of gluconeogenic enzymes. J Biol Chem. 287:25602-25614.

Mikolajka A, Yan X, Popowicz GM, Smialowski P, Nigg EA, Holak TA (2006) Structure of the N-terminal domain of the FOP (FGFR1OP) protein and implications for its dimerization and centrosomal localization. J Mol Biol. 359:863-875.

Moore MS, Blobel G (1994) A G protein involved in nucleocytoplasmic transport: the role of Ran. Trends Biochem Sci. 19:211-216.

Nakamura, M. Masuda H, Horii J, Kuma K, Yokoyama N, Ohba T, Nishitani H, Miyata T, Tanaka M, Nishimoto T (1998) When overexpressed, a novel centrosomal protein, RanBPM, causes ectopic microtubule nucleation similar to gamma-tubulin. J Cell Biol. 143:1041-1052.

Oberoi J, Fairall L, Watson PJ, Yang JC, Czimmerer Z, Kampmann T, Goult BT, Greenwood JA, Gooch JT, Kallenberger BC, Nagy L, Neuhaus D, Schwabe JW (2011) Structural basis for the assembly of the SMRT/NCoR core transcriptional repression machinery. Nat Struct Mol Biol. 18:177-184.

Regelmann J, Schüle T, Josupeit FS, Horak J, Rose M, Entian KD, Thumm M, Wolf DH (2003) Catabolite degradation of fructose-1,6-bisphosphatase in the yeast Saccharomyces cerevisiae: a genome-wide screen identifies eight novel GID genes and indicates the existence of two degradation pathways. Mol Biol Cell 14:1652-1663.

Salemi LM, Loureiro SO, Schild-Poulter C (2015) Characterization of RanBPM molecular determinants that control its subcellular localization. PLoS ONE 10:e0117655.

Santt O, Pfirrmann T, Braun B, Juretschke J, Kimmig P, Scheel H, Hofmann K, Thumm M, Wolf DH (2008) The yeast GID complex, a novel ubiquitin ligase (E3) involved 
in the regulation of carbohydrate metabolism. Mol Biol Cell 19:3323-3333.

Sazer S, Dasso M (2000) The ran decathlon: multiple roles of Ran. J Cell Sci. 113:1111-1118.

Shieh HL, Chen Y, Brown CR, Chiang HL (2001) Biochemical analysis of fructose-1,6-bisphosphatase import into vacuole import and degradation vesicles reveals a role for UBC1 in vesicle biogenesis. J Biol Chem. 276:1039810406.

Snowdon C, Hlynialuk C, van der Merwe G (2008) Components of the Vid30c are needed for the rapamycin induced degradation of the high-affinity hexose transporter Hxt7p in Saccharomyces cerevisiae. FEMS Yeast Res. 8:204-216.

Soni S, Bala S, Gwynn B, Sahr KE, Peters LL, Hanspal M (2006) Absence of erythroblast macrophage protein (Emp) leads to failure of erythroblast nuclear extrusion. J Biol Chem. 281:20181-20189.

Stone SL, Hauksdóttir H, Troy A, Herschleb J, Kraft E, Callis J (2005) Functional analysis of the RING-type ubiquitin ligase family of Arabidopsis. Plant Physiol. 137:13-30.

Suresh B, Ramakrishna S, Baek KH (2012) Diverse roles of the scaffolding protein RanBPM. Drug Discov Today 17:379-387.

Suresh B, Ramakrishna S, Kim YS, Kim SM, Kim MS, Baek KH (2010) Stability and function of mammalian lethal giant larvae-1 oncoprotein are regulated by the scaffolding protein RanBPM. J Biol Chem. 285:35340-35349.

Suzuki T, Ueda A, Kobayashi N, Yang J, Tomaru K, Yamamoto M, Takeno M, Ishigatsubo Y (2008) Proteasome-dependent degradation of alpha-catenin is regulated by interaction with ARMc8alpha. Biochem $\mathrm{J}$. 411:581-591.

Tomaru K, Ueda A, Suzuki T, Kobayashi N, Yang J, Yamamoto M, Takeno M, Kaneko T, Ishigatsubo Y (2010) Armadillo Repeat Containing alpha Binds to HRS and Promotes HRS Interaction with Ubiquitinated Proteins. Open Biochem J. 4:1-8.
Tomaštíková E, Cenklová V, Kohoutová L, Petrovská B, Váchová L, Halada P, Kočárová G, Binarová P (2012) Interactions of an Arabidopsis RanBPM homologue with LisH-CTLH domain proteins revealed high conservation of CTLH complexes in eukaryotes. BMC Plant Biol. 12:83.

Tong AH, Drees B, Nardelli G, Bader GD, Brannetti B, Castagnoli L, Evangelista M, Ferracuti S, Nelson B, Paoluzi S, Quondam M, Zucconi A, Hogue CW, Fields S, Boone C, Cesareni G (2002) A combined experimental and computational strategy to define protein interaction networks for peptide recognition modules. Science 295:321-324.

Valiyaveettil M, Bentley AA, Gursahaney P, Hussien R, Chakravarti R, Kureishy N, Prag S, Adams JC (2008) Novel role of the muskelin-RanBP9 complex as a nucleocytoplasmic mediator of cell morphology regulation. J Cell Biol. 182:727-739.

Wang D, Li Z, Messing EM, Wu G (2002) Activation of Ras/Erk pathway by a novel MET-interacting protein RanBPM. J Biol Chem. 277:36216-36222.

Winter D, Vinegar B, Nahal H, Ammar R, Wilson GV, Provart NJ (2007) An "Electronic Fluorescent Pictograph" browser for exploring and analyzing large-scale biological data sets. PLoS ONE 2:e718.

Wu Y, Sun X, Kaczmarek E, Dwyer KM, Bianchi E, Usheva A, Robson SC (2006) RanBPM associates with CD39 and modulates ecto-nucleotidase activity. Biochem J. 396:2330. 